\title{
An exploration of human services system contacts prior to suicide in South Carolina: an expansion of the South Carolina Violent Death Reporting System
}

\author{
M A Weis, C Bradberry, L P Carter, J Ferguson, D Kozareva
}

Injury Prevention 2006;12(Suppl II):ii17-ii21. doi: 10.1136/ip.2006.012427

See end of article for authors' affiliations

\section{Correspondence to:} Ms M A Weis, South Carolina Department of Health and Environmental Control, Division of Injury and Violence Prevention, 1751 Calhoun Street Columbia, SC 29201, USA; weisma@dhec.sc. gov

Accepted 3 August 2006

\begin{abstract}
Objective: To link South Carolina Violent Death Reporting System (SCVDRS) data with state government human services databases, enabling expanded analysis of suicide in South Carolina and providing a model for other jurisdictions.

Design: The SCVDRS database compiles data from vital statistics, coroner reports, and law enforcement incident and supplemental reports. The Office of Research and Statistics, South Carolina Budget and Control Board (ORS) created a "Data Warehouse", to which a variety of state agencies and healthcare providers submit data on a regular basis. A unique identifier was used to link SCVDRS data to the Data Warehouse so that data may be analyzed on aggregate and case-specific levels. Year 2004 suicide data from SCVDRS were linked to South Carolina Uniform Billing codes from hospital in-patient and emergency room billing records, State Department of Mental Health service records, and criminal justice databases. Results: SCVDRS year 2004 suicide data are augmented by hospitalization and emergency room visit data and diagnoses; State Department of Mental Health service provision; and criminal involvement. Of the 491 suicides occurring in 2004, 282 linked with hospitalization and emergency room data, 196 linked with criminal history databases, and 91 had previous contact with the State Department of Mental Health. Conclusions: Linking SCVDRS data to additional human services databases enables greater examination of factors surrounding suicide. Results show the positive benefits of partnerships created through SCVDRS, illustrate how SCVDRS and human service databases may augment each other, and suggest practitioners should explore implementation of prevention programs in specific settings.
\end{abstract}

$\mathrm{S}$ uicide in South Carolina (SC), a state in the US, is a major public health problem. From 1999-2004, 2782 SC residents commited suicide. ${ }^{1}$ Suicide is one of the top five causes of death among SC residents aged 10-34 years, and is the third leading cause of death of those aged 18-24 years. ${ }^{1}$ In $2004,56.8 \%$ of all SC violent deaths were suicides. ${ }^{2}$

To provide state and other stakeholders with timely and accurate data about violent death for use in prevention efforts, the SC Department of Health and Environmental Control (DHEC) participates in the National Violent Death Reporting System (NVDRS). The NVDRS, developed by the Centers for Disease Control and Prevention (CDC), is a statebased surveillance system compiling data from death certificates, medical examiner/coroner files, law enforcement records, and crime laboratories for resident and occurrent suicide, homicide, unintentional firearm, legal intervention, undetermined, and terrorism deaths identified through use of 10th revision of the International Classification of Diseases codes (ICD-10). ${ }^{3}$ Before the development of the NVDRS, a uniform method to systematically integrate information from individual data sources such as death certificates and coroner reports did not exist. Traditional individual sources of data did not provide all the information needed to accurately assess the factors surrounding violent deaths; for example, death certificates cannot link victim and suspect information. ${ }^{3}$ In SC the program is referred to as the South Carolina Violent Death Reporting System (SCVDRS).

The SCVDRS collects information from death certificates, coroner reports, and law enforcement reports. Data collection began with year 2003 deaths. The SCVDRS increases the amount of vital data, such as circumstance information, needed to guide suicide prevention efforts.
As a direct result of partnerships developed and strengthened through the creation and implementation of the SCVDRS, SC is able to link SCVDRS data to other health and human services databases. This linkage enables greater examination of factors of interest to practitioners in suicide prevention. For the calendar year 2004, SCVDRS suicide victim data were linked to: hospitalization and emergency room visits; diagnoses and services received from the South Carolina Department of Mental Health (DMH); and criminal justice databases to determine extent of criminal involvement. This initial analysis demonstrates the ability to link diverse databases to expand the SCVDRS and allow for additional data exploration.

\section{DESIGN}

SCVDRS incidents are initiated through use of ICD-10 codes. Trained coders manually abstract death certificates, coroner, and law enforcement reports for entry into the SCVDRS database. The CDC provides database software. Additional information regarding NVDRS methodology is available through Paulozzi et al. ${ }^{3}$ MicroSoft SQL Server is used to

Abbreviations: $C D C$, United States Centers for Disease Control and Prevention; DHEC, South Carolina Department of Health and Environmental Control; DJJ, South Carolina Department of Juvenile Justice; DUI, driving under the influence of alcohol or other drugs; DMH, South Carolina Department of Mental Health; ICD-10, International Classification of Diseases-10th revision; NVDRS, National Violent Death Reporting System; ORS, South Carolina Budget and Control Board, Office of Research and Statistics; SC, South Carolina; SCVDRS, South Carolina Violent Death Reporting System; SLED, South Carolina Law Enforcement Division; UB-92, The Uniform Billing code, specific to South Carolina, developed in 1982 by the South Carolina Hospital Association. 
select variables from the SCVDRS database to be imported into an appropriate statistical package for analyses.

A variety of state agencies and private healthcare providers submit data on a regular basis to the Office of Research and Statistics, South Carolina Budget and Control Board (ORS). Agencies retain control of their data by providing permission for other entities to access their data. The resulting network of linked databases is called the "Data Warehouse" (fig 1).

The Data Warehouse employs a unique tracking client-level identifier so that data may be analyzed on aggregate and case-specific levels. This unique tracking identifier is a randomly assigned, system generated number and is not associated with any other identifier (for example, social security number, driver's license number, telephone number, date of birth, etc). To generate this tracking number, individual identifiers on each data record are compared with identifiers on records already in the system and assigned a weighted score based on how closely the identifiers match. When the total match score between two records reaches a predefined threshold, the records are considered a match, the tracking identifier from the system record is applied to the new record, and the new record is stored within the system for use in later comparisons. If more than one comparison surpasses the threshold, the record with the highest match score is considered the match. If no match is found, the new record is assigned a new unique random tracking identifier and then stored. An individual retains the unique identifier throughout the system, allowing for the tracking of individuals across state agencies and other organizations that provide client-level data to the Data Warehouse. One benefit of this system is that it can be used to determine the array of services that an individual may be receiving from many different providers.

Through a contract with ORS, the DHEC provided a copy of a SCVDRS datafile to ORS to link SCVDRS deaths to the databases that are maintained in the Data Warehouse. Specifically, SC year 2004 suicide deaths were linked with several databases and cases tracked to determine a variety of measures and system contacts as detailed below.

\section{Uniform billing codes from hospital in-patient and emergency room visits}

In 1982 the South Carolina Hospital Association developed a hospital uniform billing coding manual (now called the UB92), which was adapted from the American Hospital Association's National Uniform Billing Manual. The UB-92 contains codes that are specific to SC. These codes allow consistent analysis of hospital and emergency room services and charges; ORS holds the database of the billing data. The SCVDRS datafile was linked to UB-92 hospital in-patient and emergency room records from January 2003 through December 2004. The resulting file was then analyzed for a variety of measures including: number of inpatient hospitalizations and emergency room (ER) visits, E-code (that is, the mechanism of injury) of last hospital or emergency room encounter prior to suicide, number of days between last hospital or emergency room encounter and suicide, and E-code designation that hospital or emergency room contact was the result of a suicide attempt.
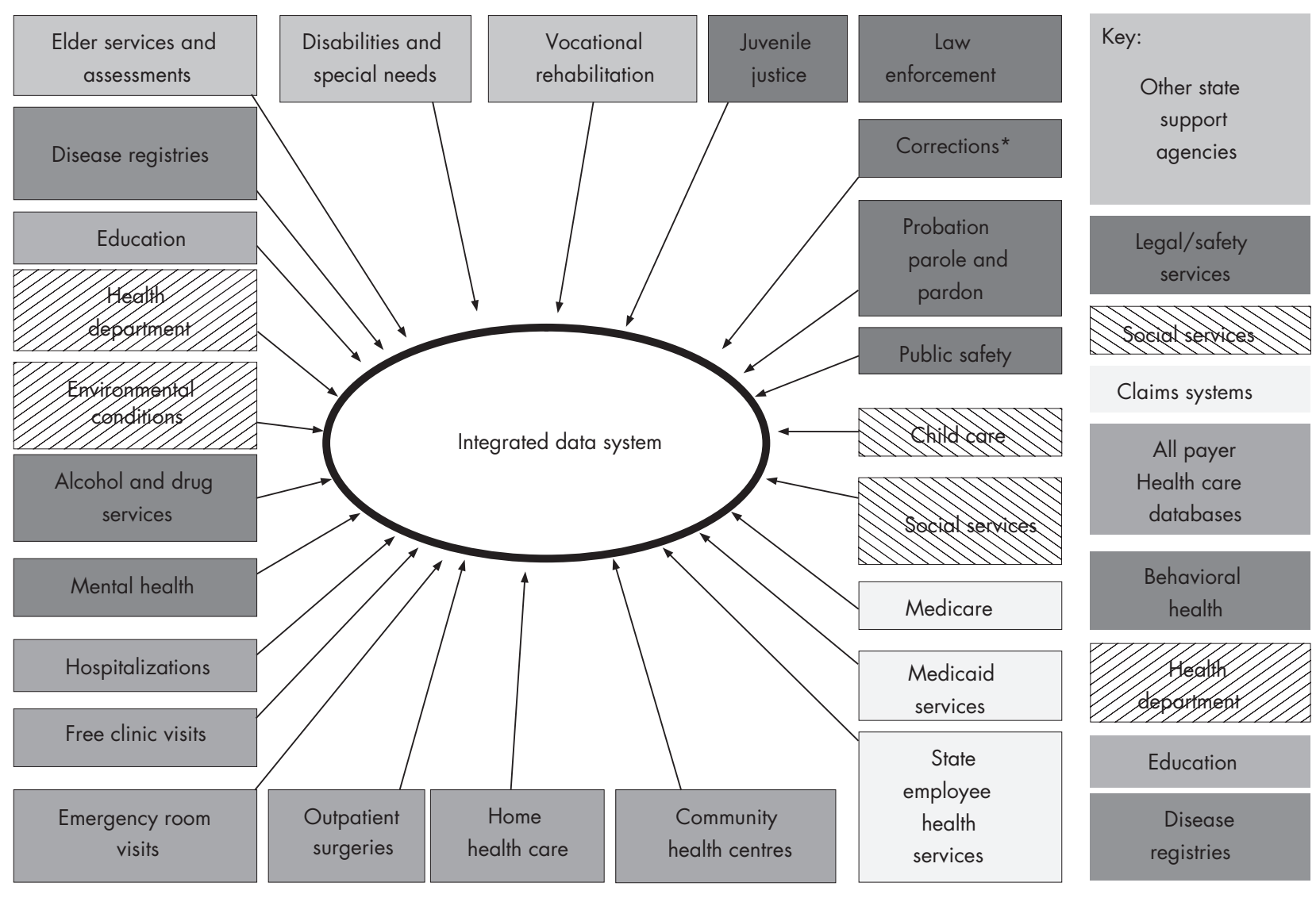

*Still in contract negotiations.

Figure 1 The Data Warehouse. 


\section{South Carolina Department of Mental Health}

The ORS, though contract with the DHEC, facilitated release of Department of Mental Health (DMH) data to be linked with the SCVDRS file. The ORS matched year 2004 suicides to DMH records (beginning January 1996) to determine DMH contact prior to suicide, diagnosis, and the time interval between last date of service by the DMH and date of death.

\section{State Law Enforcement Division and State Department of Juvenile Justice}

Through a contract with the ORS and approval of data release by the State Law Enforcement Division (SLED) and Department of Juvenile Justice (DJJ), the SCVDRS year 2004 suicide deaths were matched with the SLED and DJJ histories. The SLED database was automated in 1977, comprising all arrests in and after that year. Individuals who were arrested on or after 1977 have their entire criminal histories automated (paper files of arrests before 1977 were entered into the database). If an individual was arrested before 1977, but not during or after 1977, that arrest is not part of the SLED automated system. The DJJ database was automated in 1983. When the matching was completed, an itemized file was provided to SCVDRS. Records were reviewed for all arrests and arrests relating to criminal domestic violence, driving under the influence of alcohol, and sex crimes (sexual assault, indecent exposure) occurring 018 months prior to suicide completion. SCVDRS narrative data (abstracted from coroner reports and local law enforcement incident and supplemental reports) were then reviewed to determine if appropriate arrest and criminal history data were captured by the SCVDRS.

\section{RESULTS}

Over half $(56.8 \%, \mathrm{n}=491)$ of the 2004 violent deaths $(\mathrm{n}=864)$ reported via SCVDRS are suicides. This represents 1.3 completed suicides per day, or a rate of 11.7 per 100000 population. Demographically, the majority of victims $(70.7 \%)$ were white males, followed by white females (16.7\%), black and other males (10.2\%) and black and other females $(2.4 \%)$. Hispanic ethnicity was not included in this analysis. Males aged 75-84 years had the highest rate of suicide death $(38.3$ per 100000 ), while males aged 45-54 demonstrated the highest number of deaths $(\mathrm{n}=88)$. Twenty victims were aged 17 years and younger. Among victims aged 25 years and older $74.2 \%$ completed a high school education.

The successful linkage of the SCVDRS data to additional human service databases provides supplementary insight to SCVDRS findings. The current analysis is designed to provide a broad overview; demographic differences in the findings are an area for later exploration.
UB-92 hospital in-patient and emergency room visits Of the 491 suicides in 2004, 282 cases $(57.4 \%)$ were linked to year 2003 and 2004 UB-92 hospital discharge and emergency room records (fig 2). Two hundred and eight individuals (42.4\%) did not link; 207 of these individuals did not have a hospitalization or emergency room visit during 2003-04, and a unique identifying number was unable to be assigned to one individual. The one individual for whom a unique identifier could not be assigned lacked sufficient identifying information (for example, date of birth, social security number, race, sex, or a combination of these).

From January 2003 through December 2004, 282 individuals logged a total of 865 visits to a hospital or emergency room. Emergency room visits accounted for 573 visits (223 persons), and in-patient care accounted for 292 visits (158 persons). Individuals averaged three visits to a hospital within the two years prior to suicide completion (table 1).

Primary and secondary E-codes indicate intention. From the 282 linked files, 160 (56.7\%) individuals had primary or secondary injury E-codes from the final hospital or emergency room visit prior to suicide. Injuries were identified as suicide attempts in 80 hospital encounters by 70 persons (24.8\% of linked records). Thirty eight $(47.5 \%)$ encounters were firearm related, and $35(43.8 \%)$ were poison related.

For 68 individuals ( $24.1 \%$ of linked files), less than one day elapsed between the last hospital encounter and suicide completion. It is possible this number reflects those who died upon arrival, eliminating the possibility that prevention programs would prevent a later suicide. Approximately $7 \%$ $(7.1 \%, \mathrm{n}=20)$ of completed suicides occurred within $1-7$ days of the final hospital encounter, and $16.7 \%(n=47)$

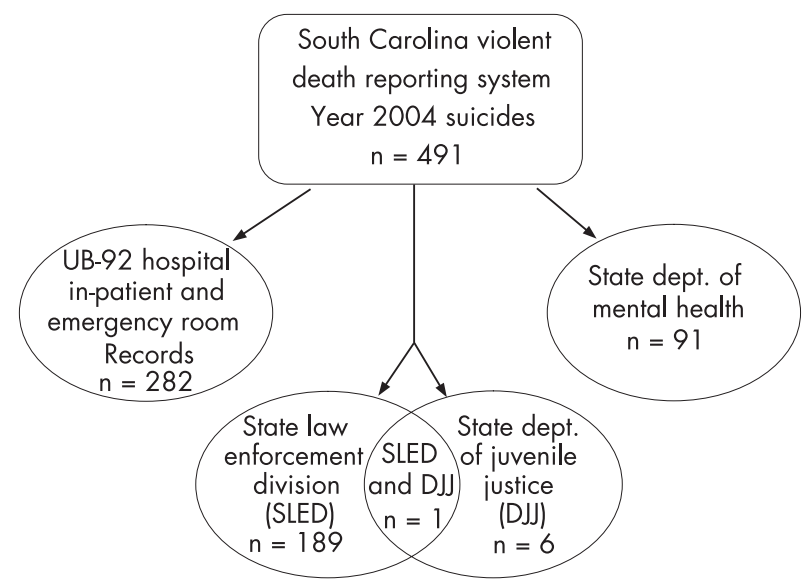

Figure 2 SCVDRS data linkage.

Table 1 Number of South Carolina in-patient hospitalizations and emergency room visits for persons who completed suicide in 2004, January 2003-December 2004 (282 linked individuals)

\begin{tabular}{lllll}
\hline $\begin{array}{l}\text { Year of hospital } \\
\text { encounter }\end{array}$ & Type of encounter & Encounters, $\mathbf{n}$ & Persons, $\mathbf{n}$ & $\begin{array}{l}\text { Visits per } \\
\text { person, } \mathbf{n}\end{array}$ \\
\hline $2003 \& 2004$ & Total & 865 & 282 & 3.07 \\
& Emergency room & 573 & 223 & 2.57 \\
2003 & Inpatient & 292 & 158 & 1.85 \\
& Total & 462 & 189 & 2.44 \\
2004 & Emergency room & 311 & 147 & 2.12 \\
& Inpatient & 151 & 93 & 1.62 \\
& Total & 403 & 188 & 2.14 \\
& Emergency room & 262 & 140 & 1.87 \\
& Inpatient & 141 & 95 & 1.48 \\
\hline
\end{tabular}


Table 2 South Carolina Year 2004 suicide victims with Department of Mental Health (DMH) contact within 6 months prior to death*

\begin{tabular}{lccc}
\hline Time & $\begin{array}{c}\text { Number } \\
\text { of victims victims }\end{array}$ & $\begin{array}{c}\text { Cumulative } \\
\text { number of }\end{array}$ & $\begin{array}{l}\text { Percent of total } \\
\text { victims with DMH } \\
\text { contact }\end{array}$ \\
\hline 1 day & 2 & 2 & 2.2 \\
2 days & 0 & 2 & 2.2 \\
3 days & 2 & 4 & 4.4 \\
4 days & 1 & 5 & 5.5 \\
5 days & 1 & 6 & 6.6 \\
6 days & 1 & 7 & 7.7 \\
1 week & 7 & 14 & 15.4 \\
2 weeks & 4 & 18 & 19.8 \\
1 month & 11 & 29 & 31.9 \\
2 months & 7 & 36 & 39.6 \\
3 months & 4 & 40 & 44.0 \\
4 months & 2 & 42 & 46.2 \\
5 months & 3 & 45 & 49.5 \\
6 months & 1 & 46 & 50.5 \\
Total, $0-6$ months & 46 & & \\
\hline *Ninety one individuals had a DMH record between 1996 and death. \\
\hline
\end{tabular}

occurred within 1-30 days of the final hospital encounter. The median number of days between the final hospital encounter and suicide (excluding those with less than one day) is 110 . The 25 th and 75 th percentiles are represented by 35 and 271 days, respectively.

\section{Department of Mental Health}

History of contact with the DMH and year 2004 suicide victims between January 1996 and the date of suicide was reviewed. Electronic DMH records before 1996 were not available. Ninety one suicide victims were served by the DMH during this time period, representing $18.5 \%$ of SC suicides in 2004. The number of service contacts (that is, visits) with the DMH ranged from one $(n=12)$ to more than $800(n=1)$, with $75.8 \%(n=69)$ of victims having between one and 34 visits. The median number of visits per patient is 8 . The 25 th and 75th percentiles are represented by 3 and 34 visits, respectively.

The time interval between the last date of service contact with the DMH and suicide was analyzed (table 2). Two victims had contact with the DMH within one day of suicide; seven victims had DMH contact in the six days before suicide; and 46 victims had contact with the $\mathrm{DMH}$ within six months of suicide.

Primary diagnosis of victims at time of last visit varies. Depression was most common $(\mathrm{n}=31)$, followed by "other mental illness" $(n=15)$ and schizophrenia $(n=12)$. "Other mental illness" are those not classified as attention deficit/

Table 3 South Carolina Year 2004 suicide victims with criminal justice system contact within 18 months prior to death* $^{*}$

\begin{tabular}{lcccc}
\hline & $\begin{array}{l}\text { Criminal } \\
\text { domestic } \\
\text { violence }\end{array}$ & DUIt & Sex crime & Total \\
\hline 1 week & 1 & 0 & 2 & 3 \\
1 month & 3 & 3 & 0 & 6 \\
3 months & 2 & 0 & 1 & 3 \\
6 months & 2 & 3 & 0 & 5 \\
12 months & 4 & 2 & 1 & 7 \\
18 months & 4 & 1 & 0 & 5 \\
Total, 0-18 months & 16 & 9 & 4 & 29 \\
Ever had contact & 40 & 82 & 9 & 131 \\
\hline
\end{tabular}

*196 individuals had an arrest record of some type.

†Driving under the influence of alcohol or drugs. hyperactive disorder, anxiety, bipolar, conduct, depression, post-traumatic stress syndrome, substance abuse/dependence, borderline personality disorder, co-occurring diagnoses, and other personality disorders.

\section{Criminal justice: SLED and DJJ}

Criminal histories were searched for all 491 suicide victims. The automated SLED database contains all arrests in and after 1977, and arrests before 1977 of those with an arrest in or after 1977. The DJJ database contains arrests in and after 1983. One hundred and ninety six individuals had a criminal history, representing $39.9 \%$ of year 2004 suicides (fig 2). Six individuals had a juvenile arrest record, and 189 had an adult arrest record. One individual had both a juvenile and an adult arrest record. Sixty nine individuals $(35.2 \%$ of those with a criminal history) were arrested within 18 months prior to suicide completion, including all six individuals in the DJJ database and the individual in the SLED and DJJ databases (table 3).

\section{Criminal domestic violence arrests}

Forty individuals $(20.4 \%$ with a criminal history) had an arrest record for criminal domestic violence. Sixteen of those individuals, or $40.0 \%$, had criminal domestic violence arrest records $0-18$ months prior to suicide. Two of these individuals, or 5\%, had more than one arrest during the time period. Of those 16 cases, SCVDRS narratives indicated that only one law enforcement report mentioned a prior arrest or status as a suspect in a criminal domestic violence case. However, one law enforcement report and one coroner report mentioned criminal domestic violence as relevant to the suicide (that is, "Victim had physically attacked girlfriend"). In one case both the law enforcement report and coroner report indicated that the suicide victim had been a suspect in the death of his girlfriend.

\section{Driving under the influence of alcohol or other drugs arrests}

Eighty two individuals, or $41.8 \%$, with a criminal history had arrest records for DUI. Nine of these individuals, or $11.0 \%$, had arrests in the 18 month period prior to suicide. Three individuals, or $3.7 \%$, had an arrest within the month of death, and five individuals, or $6.1 \%$, between 6-12 months prior to death. Narrative information from law enforcement and coroner reports did not reflect DUI in any case. However, comments such as "Victim was known to have a drinking problem," were present.

\section{Sex crimes arrests (indecent exposure, sexual assault, etc)}

Nine individuals with a criminal history, or $4.6 \%$, had ever been arrested for a sex crime. Four of these individuals, or $44.4 \%$, had been arrested for a sex crime within 18 months prior to death. Two individuals, or $22.2 \%$, completed suicide within a week of arrest. One individual, or $11.1 \%$, was arrested twice within the week prior. In only one case did the law enforcement and coroner narrative mention the arrests.

\section{CONCLUSIONS}

The effort of linking SCVDRS data with other health and human services databases resulted in a greater understanding of the factors surrounding suicide. Findings may be directly applied to prevention programs and efforts to improve health and human service systems. For example, linkage with hospital data shows $16.7 \%(n=47)$ of year 2004 suicide victims died within 30 days of a final hospital encounter. This result suggests a potentially effective time and place to implement a suicide prevention program. A support infrastructure for increased suicide prevention 
strategies is being built. SCVDRS data have already been used in the South Carolina Suicide Prevention State Plan, to initiate dialogue between non-traditional partners and in grant applications by stakeholders.

\section{UB-92 in-patient hospitalizations and emergency room visits}

Results showed that in the last visit before a completed suicide, injuries were identified as suicide attempts in 80 hospital encounters by 70 persons. The number of individuals with a previous suicide attempt as indicated by hospital inpatient and emergency room visits reflects visits from January 2003 to December 2004 only. Visits to primary care physicians are not reflected. Therefore, the 70 persons is probably an undercount. In contrast, SCVDRS circumstance data show that 45 individuals had a "history of attempting suicide". ${ }^{2}$ The disconnect possibly results from family and friends being unaware or not sharing this history with coroner and law enforcement personnel investigating the death. SCVDRS data are critical for timely surveillance, but the addition of UB-92 data provides additional knowledge.

\section{Department of Mental Health}

Ninety one of the SC year 2004 suicide victims were served by the DMH, representing $18.5 \%$ of SC suicides in 2004. This number reflects only those victims who were served by the $\mathrm{DMH}$ and does not include treatment from private doctors, counselors, or facilities.

SCVDRS circumstance data, available for 371 (75.6\%) of the suicide victims, indicate 80 individuals "had a history of treatment for mental illness" and 78 victims were "currently receiving mental health treatment" at the time of death. "Ever treated" and "currently receiving" treatment include both public (DMH) and private services. SCVDRS circumstance data are dependent on the information received and reported by law enforcement and coroners. Linkage of DMH data adds a layer of understanding by illustrating a greater number of suicide victims had "ever" received mental health treatment; conversely SCVDRS, by including information from the investigation beyond DMH services, provides more inclusive current mental health treatment information.

\section{Criminal justice: SLED and DJJ}

A total of 131 individuals (26.7\% of all year 2004 suicides) had a criminal justice contact for criminal domestic violence, DUI, or a crime in the 18 months before suicide. The linkage illustrates the potential of linking SCVDRS law enforcement and coroner report data with criminal justice databases. Though a causal relation between an arrest in the 18 months before suicide and the completed suicide act cannot be determined by the current analysis, the context of suicide following an arrest of these types, and potential for prevention programming, is something to be explored further.

The narrative data provided by SCVDRS adds depth to comparison by revealing circumstances surrounding the immediate event. In several cases a previous criminal domestic violence arrest was revealed through the criminal justice database, and SCVDRS revealed that the suicide occurred in relation to an immediate criminal domestic violence event not reflected in arrest data. Criminal justice databases should be used in determining a criminal history of a victim, while SCVDRS excels at providing data surrounding the immediate event.

The initial statistical results are exciting for SC. SCVDRS data have been enhanced and have shown potential for

\section{Key points}

- The South Carolina Violent Death Reporting System (SCVDRS) provides previously unavailable violent death data.

- The SCVDRS, in addition to providing violent death surveillance, fosters partnerships among state agencies. This may be replicated by other jurisdictions nationally and internationally.

- SCVDRS data may be supplemented through linkage with criminal justice and health and human services databases.

- The SCVDRS and the Data Warehouse provide separate, but complementary, data regarding victims of violent death. Jurisdictions may incorporate similar systems to increase their knowledge regarding violent death.

practical application by practitioners. SC's experience demonstrates how violent death surveillance may be used beyond the initial system. The technical aspects show the possibilities to other jurisdictions throughout the world. However, beyond the numbers and program codes produced is the key to the process and greatest lesson to be demonstrated. Through SCVDRS, SC has created and nurtured partnerships among agencies and program collaborators that made it possible to expand into other avenues. SC's experience in intra- and interagency collaboration in creating the Data Warehouse, and linking with SCVDRS data, may serve as an example for other states and countries.

\section{ACKNOWLEDGEMENTS}

This work was supported by the Centers for Disease Control and Prevention (CDC), National Violent Death Reporting System Grant No \#U17/CCU422396. Individuals contributing to development of the SCVDRS, ORS Data Warehouse and data analysis, and this article: Randy Rambo, ORS; Wesley Gravelle, DHEC; Nikolay Lipsky, CDC.

\section{Authors' affiliations}

M A Weis, South Carolina Department of Health and Environmental Control, Division of Injury and Violence Prevention, Columbia, SC, USA C Bradberry, Office of Research and Statistics, South Carolina Budget and Control Board, Columbia, SC, USA

L P Carter, Division of Injury and Violence Prevention, South Carolina Department of Health and Environmental Control, Columbia, SC, USA J Ferguson, Office of Public Health Statistics and Information Services, South Carolina Department of Health and Environmental Control,

Columbia, SC, USA

D Kozareva, Division of Biostatistics and Health GIS, South Carolina Department of Health and Environmental Control, Columbia, SC, USA

The findings and conclusions in this report are those of the authors and do not necessarily represent the views of the Centers for Disease Control and Prevention.

\section{REFERENCES}

1 South Carolina Community Assessment Network. Available at http:// scangis.dhec.sc.gov/scan/ (accessed September 2006).

2 South Carolina Department of Health and Environmental Control. South Carolina Violent Death Reporting System: 2004 Violent Deaths in South Carolina-Preliminary Report. Released September, 2004.

3 Paulozzi LJ, Mercy J, Frazier Jr L, et al. CDC's National Violent Death Reporting System: background and methodology. Inj Prev 2004;10:47-52. 